\title{
Physics in Medical Science
}

\author{
Parashu Ram Poudel \\ Department of Physics,PN Campus,Pokhara \\ parashupoudel@hotmail.com
}

\begin{abstract}
The domain of Physics covers vast area of scientific knowledge. Basic research on assemblies of atomic or nuclear radiation and gyromagnetic moments led to powerful technique for studying molecular structure as well as solid lattices. It led to invention and development of modern medical diagnostic and theraputic tools which have revolutionized the medical practices. Advancement in medical researches as seen today will be well-nigh impossible without the use of the finding of Physics. The funding made on Physics is in fact another way of funding made on human health.
\end{abstract}

Keywords: radioactivity, crystallography, radioimmune assay, MRI, CAT, PET.

\section{Introduction}

Physics is the science which deals with the study of nature and natural phenomena. It is the most fundamental of all the sciences. The realms of physics span distance from the sub nuclear world to the whole of the cosmos, and time from less than a billionth of a trillionth of a second to the age of the universe. To study phenomena across these epochal scales, to devise experimental tools that provide more powerful means for viewing nature and to create theories that allow us to comprehend what has been seen, are the goals and the achievements of physics. In deepening our view of nature, physics has profoundly affected our view because of the underlying assumption of Physics that there is an order in the natural world and that the human mind can understand that order. By generating new technologies and contributing neighboring sciences Physics has helped to transform our daily life, permitting a comfort and freedom of action. In the past even in the technically advanced nations, people devoted most of their energy to securing food and shelter. Physics has done much to mould the shape of modern society. The search to understand elementary phenomena has led to expanded views of all nature and to miraculous inventions. Thus, the path of this search is unpredictable.

Physics has played a key role in the advancement of medical science. Medical science has been revolutionized by the discoveries in Physics in the past. A series of advances in Physics has a decisive and direct impact on medical science Today nearly all the diagnostic and therapeutic tools of modern hospitals have their origin in Physics research.

\section{Applications of Physics in Medical Science}

\section{Understanding the Body at Molecular Level}

The basis for medical practice resides in an nderstanding of how the body functions. Body works as a result of aggregation of function of the biological molecules. Physics has contributed at the level of the molecular structure and its functions. As an example, proteins are giant molecules which provide cells with their internal mechanical support. They are also responsible for all the chemical processes at the core of the life. Biophysicists have determined exact atomic structure of proteins by X-ray crystallography (the technique for which W L Bragg and W H Bragg won the Nobel Prize in Physics in 1915) or by accelerator technology (A O Lawrence received the Nobel Prize of Physics in 1939 for inventing the first of these accelerator, the cyclotron). Understanding the structure of complex biological molecules is the key to developing new pharmaceuticals. As for example, a virus enters a cell by penetrating the cell membranes after docking to a surface protein by a process that can occur only when the shape of the virus fits a lock. Determining the underlying structure of a virus such as common cold virus or HIV opens the possibility of developing pharmaceutical molecules that compete with these viruses at the docking sites and thus prevent viral penetration into the cell.

Most drugs work by binding to unique sites on specific protein and modifying how the protein does its job. The search for molecules that can bind selectively to proteins and alter their function is very difficult and insufficient if done by trial and error. It is the 
application of Physics techniques such as x-rays crystallography that allows determining the structure of viruses and identifying sites on a virus with a therapeutic molecule. Design and development of such drugs are done by the physicists and is now a major aspect of research.

Knowing the structure of proteins is essential for understanding their functions. Cells contain tiny molecular motors which are the family of proteins that help the cell to move or the material within the cell from one place to another. The most familiar example of these motors is muscle contraction and the pumping of the heart. Molecular motors are essential for the job performed by the cells.

The beating of the heart and function of the brain require another kind of protein that produces electrical signal in cell. The Electro Cardiogram (ECG) and Electro Encephalogram (EEG) are manifestations of the operations of proteins and are used for the diagnosis of heart and neurological diseases. Biophysicists have also learned how these proteins generate their electrical signals and this knowledge has given us many therapies ranging from drug to treat abnormal heart beats to the treatment of epilepsy.

\section{Diagnosis}

Physics plays an ever more important role in diagnosis. The use of ionizing radiation has been important therapeutically for more than half a century. Labeling red blood cells with radioactive isotopes of chromium permits the lifetime of these cells to be measured and this method can be used to determine if anemia is the result of decreased production or increased destruction of red blood cells. The radioactive assay makes use of antibodies that have been made radioactive: these antibodies detect minute quantities of hormones and other chemicals by binding to them and providing a radioactive tag that can be used to detect the presence of the molecule. Because signaling molecules, like hormones, are present at minute concentrations in the blood, Yalow received the Nobel Prize in 1977 for the development of technique, the radio immune assay. Other bodily fluids, some organs specifically take up certain atoms or chemical compounds and this fact has enabled physicians to assess organs' function as to identify the pressure of damage by monitoring the uptake of these substances that have been tagged with radioactivity.

$\mathrm{X}$-rays of electromagnetic radiations with extremely short wave lengths were discovered by Wilhelm Conrad Roentgen, a
German professor of physics, in his lab on Nov 8, 1895 which has been found to be remarkable medical achievement. For this descovery Roentgen won the first Nobel in Physics in the year 1901. This enables us to look inside a living body noninvasively. The passage of X-rays through biological tissues can be recorded with photographic films and other detectors. The analysis of X-ray images of the body is an extremely valuable medical diagnostic tool. In penetrating the tissues, the radiation is absorbed differently depending on the densities of the tissues being penetrated. The radiation emerging from tissue thus produces on a photographic film or a fluorescent screen, an image of the structure of differing densities of the tissue.

A new form of X-ray imaging computerized axial tomography (CAT scanning) during the 1970s in the USA and Britain measures the attenuation of X-rays entering the body from many different angles. Cormak received the 1979 Nobel Prize for developing computrised tomography, the standard method for extrating three dimentional information from two dimentional projection. From these measurement a computer reconstructs the organ under study in a series of cross section or planes. This technique allows soft tissues such as liver and kidney to be clearly differentiated in the images reconstructed by the computer. This procedure adds enormously to the diagnostic information that can be provided by conventional X-rays. CAT scanners are now used in many hospitals and medical centers throughout the world as standard method for extracting three dimensional information from two dimensional projection.

A still more recently developed technique is magnetic resonance imaging (MRI) in which radio waves are beamed into an individual which is subjected to a powerful magnetic field. Different atoms in the body absorb radio waves at different frequencies under influence of magnetic field. The way in which absorption takes place is measured and used by a computer to contrast image of internal structures. MRI, a creation of physicist allows the doctors to peer into the human body as clearly as they can view the surface. MRI is none perturbing, non invasive and free of any known side effect. Its role in medical diagnosis is expected to be revolutionary. MRI, the result of atomic nuclear and high energy physics is last centuries' the greatest advance in diagnosis. Isidor Isaac Rabi recieved the 1944 Nobel Prize in Physics for the discovery of magnatic resonance in nuclei. 


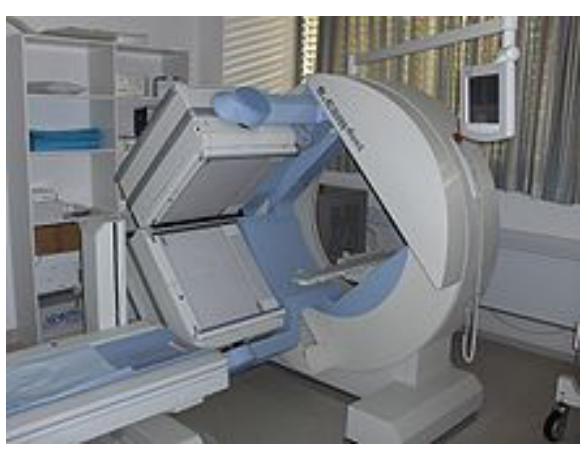

Fig.1: MRI Instrument

To understand the functioning of human brain, high resolution scanning techniques such as Functional Magnetic Resonance Imaging (FMRI) and Positron Emission Tomography (PET) are being applied. PET scanning involves the emission of particles of antimatter by compound ejected into the body being scanned. These particles, positrons, are neutralized by their opposites, electrons, and energy is released in the form of radiator as matter and antimatter annihilate each other. Detectors arranged around the body pick up the energy released and use it to follow the movements of the injected compound and its metabolism. These relatively new radiological techniques provide much safer means of examining internal body structures. They also yield precise and cleaner images for the physicians and diminish the margin of error in therapeutic measures. These techniques are sensitive to local brain metabolism or even to local concentration of complex psychoactive biochemical molecules that are thought to regulate brain function and for some forms of drug addiction. The invention of powerful computer in ultrasound has also revolutionized the imaging. Advances in imaging also extends to light microscopy. Invention of Phase Contrast Microscopy (for which Frits Zernike received Nobel Prize in 1953) improves the traditional light microscopy.

Radioisotopes, the isotopes that radiate radioactive radiation, are also used in diagnosis. The use of radio isotopes in medicine began after the discovery of artificial radioisotopes by E. Fermi in 1935. Fermy received Nobel Prize in 1938 for this discovery. In isotope scanning a radioisotope is introduced into the body, usually by means of intravenous injection. The isotope is then taken up in different amounts by different organs. Its distribution can be determined by recording the radiation it emits and through changing its concentration, it is often possible to recognize the presence, size and shape of the various abnormalities in body organs. The radiation emitted is detected by scintillation counter, which is moved back and forth over the organ being scanned. These messages can then be electronically recorded and studied by clinicians. The isotope usually has a short half life and thus decays completely before its radioactivity can cause any damage to the patient's body.

Different isotopes tend to concentrate in particular organs. For example, iodine-131 settles in the thyroid glands and can reveal a variety of defects in thyroid functioning. Another isotope carbon -14 is useful in studing abnormalities of metabolism that underlie diabetes, gout and anemia.

\section{Therapy}

Radiotherapy, the use of radiation sources in the treatment or relief of diseases, is the most important use of nuclear physics in medicine (H. Bacquerel, M. Curie and P. Curie received the Nobel Prize in Physics in 1903 for the discovery of radioactivity). Radiation therapy almost always makes use of ionizing radiation, deep tissue penetrating rays, which can physically and chemically react with diseased cells to destroy them. Radiation therapy is used for cancer and for blood disorder such as leukemia. To destroy cancerous cells with minimal damage to normal healthy tissue, the radiation may be administered to the body by implanting radioactive substances into the tumors or by exposing the body to external sources of high energy rays that penetrate internally. Ionizing radiation bombards the cells exposed to it and break the molecular bonds essential to cell growth which eradicates the cancer growth that reproduces rapidly. The radiation sources are gamma emitters which can be radium, radon or radioactive isotopes of such metals as cesium, cobalt, gold, iridium and tantalum. This type of therapy remains one of the three options for treating cancer. Either alone or in combination with surgery and chemotherapy, ionizing radiation is used as therapy for most malignancies.

The invention of LASER by T Maiman in 1960 added another brick to medicine. Since one year of its invention in 1961, LASER has been used to destroy a retinal tumor. Ophthalmology is the most benefited field where LASER is used for diagnosis as well as surgical cures. It enables doctors to preciously vaporize a tissue or shape it as they wish. A classical example is LASER surgery where LASER is used to reshape the cornea. LASER is used to crush the kidney stone in bloodless way. It is also used to treat skin tumors as well as inaccessible brain tumors. Therapeutic use of laser is 


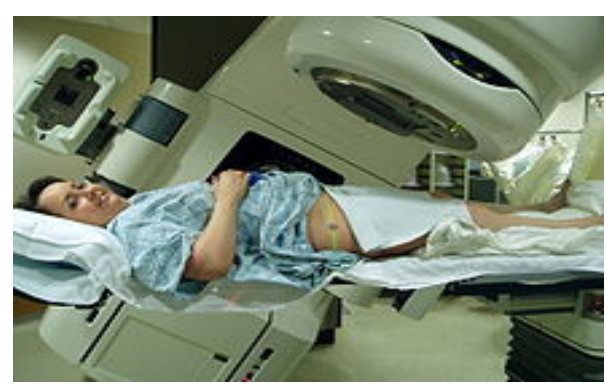

rapidly increasing.

Fig. 2: Radiation therapy of the pelvis. Lasers and a mould under the legs are used to determine exact position

Ultrasound or ultrasonic is one of the non invasive diagnostic tools from physics that has found an important place as a nonsurgical treatment of kidney stones and for cleaning of surgical instruments. It has been used to relieve pain due to arthritis and neurological pain, to restore contracted finger, to conduct bloodless surgery, to extract broken teeth and to loosen scar tissue. Ultrasonic waves are also used in sterilization of milk and water.

The invention of optical fiber by physicists is another revolution in therapy. In medical science optical fiber endoscope exemplifies the many new diagnostic instruments. With fiber optics and video imaging doctors can now see much of the inner surface of humans. A more recent trend has been to use fiber-optics imaging to permit surgery through tiny incisions. Many appendectomies and other surgical procedures even including heart surgery are now carried out in less invasive way. Because of damage caused by large incisions and the exposure of internal organs is minimized, this remote surgical procedure can be less dangerous and patients can recover more quickly and with less discomfort.

New materials have also become available to replace damaged or missing body components. Silicon is used in reconstructive surgery to provide internal support for soft tissue and hard plastic materials are transforming dentistry by permitting better reconstruction of damaged teeth. These materials not only are strong but also bound well to the tooth surface and expand with heat like the natural tooth. This means that a much wider range of tooth damage can be repaired, and that the repairs are much more durable than in the past.

\section{Conclusion}

Physics is contributing directly to the medical care by providing knowledge of the body at the molecular level with new analytical tools and diagnostic techniques. From the treatment of cornea to the diagnosis of brain diseases, application of the discoveries of physics have dominant role and hence, it revolutionizes the medical practices. Physics is addressing increasingly complex problems of medical science. In addition to basic research, it also contributes directly to the quality of medical care by providing new analytical tools, diagnostic techniques, and therapies. X-ray tomography has had a major impact on $\mathrm{x}$-ray diagnostics; magnetic resonance imaging is widely regarded as a revolutionary advance in medical diagnostics. Ultrasonic imaging is yet another of the noninvasive diagnostic tools from Physics. Lasers are finding increasingly widespread applications in medicine. Laser surgery replaces some highly delicate or traumatic operations with simple and straightforward procedures. Fiber-optic endoscopes exemplify many new diagnostic instruments. Combined with lasers, the endoscopes can be used to provide new therapies that may replace elaborate surgical procedures. Nuclear medicine and radiation therapy have much contribution to medicinal diagnosis and therapy. Inventions of such many new instruments and technologies from Physics are today enhancing the quality of health care of human beings. Physics has great impact on the advancement of medical science. The investment on Physics is also the investment on human health.

\section{References}

[1] Poudel P.,Gautam A., Adhikari B., Thapa M., Khatry M., 2009. Principle of physics. Ayam Publication, Kathmandu,Nepal

[2] John S. Rigden, 2000. Building blocks of matters. McGraw Hill, New York.

[3] National academy of sciences, Physics in New Era: An Overview, 2000, National Academy Press, Wasington,DC

[4] Alan Isaac, 1996. Dictionary of Physics. Oxford University Press, New York.

[5] Michael S.Witherel, Interdependent sciences: Physics and Medicine, http://www.fnal.gov/pub/pulse

[6] Nuclear Physics Pannel, Nuclear Physics National Academy Press, Wasington,DC

[7] Microsoft Corporation, Encarta, 2009 Microsoft Corporation

[8] Encyclopedia, Britannica, 2010

[9] Brian R. Martin, 2006. Nuclear and Particle Physics, An Introduction, John Wiley \& Sons

[10] Basic Physics of Nuclear Medicine http://en.wikibooks. org/wiki 